\title{
Preparation of Materials for EBSD using an Adjustable Broad Beam Ion Source
}

R. R. Cerchiara, P. E. Fischione, J. H. Lange, P. V. Woods, J. M. Matesa, M. F. Boccabella and A. C. Robins

E. A. Fischione Instruments, Inc., 9003 Corporate Circle, Export, PA 15632

Analysis by Electron Backscatter Diffraction (EBSD) requires that the sample surface be free of deformation, oxidation and hydrocarbon contamination. The options for creating such a surface include mechanical methods, electropolishing and ion based techniques.[1-3] Often a combination of techniques is required, ending with ion milling using either a focused (FIB) or broad (BIB) ion beam. A liquid metal $(\mathrm{Ga})$ ion source is predominant in the first case, while various inert gas (Ar, $\mathrm{Kr}$ ) ion sources have found applications in the latter case.

The advantages of the FIB approach are faster ion milling rates, site specificity at a greater instrument resolution, full automation and precise serial sectioning. The advantages of the BIB approach include lower ion beam energies, reduction of the severity of the ion - sample interactions (amorphization, implantation, curtaining), and ion milling of larger relative areas $(\sim 10 \mathrm{~mm} \mathrm{X} \sim 10$ $\mathrm{mm})$ in reasonable time. Damaged layers $(0.5-2 \mathrm{~nm}$ thick) may remain after FIB milling with $2-5$ $\mathrm{keV} \mathrm{Ga}$ ions. [4] These layers contain amorphous material and implanted Ga ions, with the distribution dependent upon the sample type and preparation protocol.

Alternative protocols which begin with mechanical sectioning, followed by grinding and lapping and finally BIB milling, will be presented. The goal of this preparation sequence is to produce a parallel - sided sample with a surface finish of less than $1 \mu \mathrm{m}$. This sample geometry is required so that tilting may occur eucentrically with respect to a BIB ion source. Precise tilting permits large surface areas to be ion milled at low incident angles, in the minimum amount of time. Reducing the processing time enhances sample quality while optimizing throughput. The use of a dry pumped vacuum system backed by a (liquid nitrogen filled) cold trap will also be assessed.

To advance this research, BIB milling was conducted in an instrument equipped with a novel Ar ion source designed with an adjustable ion beam diameter. The ion source voltage ranged from $6 \mathrm{keV}$ to $100 \mathrm{eV}$, while the ion beam current was independently variable from 10's of uA to 100's of nA. Application examples will be presented that detail the effects of decreased voltage and current as a function of the ion beam diameter. Increased sample quality is established based on qualitative improvements in SEM imaging and a quantitative enhancement of the EBSD results.

\section{References}

[1] A. J. Schwartz et al., "Electron Backscatter Diffraction in Materials Science”, Kluwer Academic / Plenum Publishers, New York, 2000.

[2] L. A. Gianuzzi et al., "Introduction to Focused Ion Beams: Instrumentation, Theory, Techniques and Practice", Springer New York, 2005.

[3] M Nowell et al., . Microanal. 11 (Suppl. 2), 2005, pp. $504-505$.

[4] L. A. Giannuzzi et al., Microsc. Microanal. 11 (Suppl. 2), 2005, pp. 828 - 829. 